Research Paper

\title{
Disruption of Wnt/ $\beta$-catenin Signaling in Odontoblasts and Cementoblasts Arrests Tooth Root Development in Postnatal Mouse Teeth
}

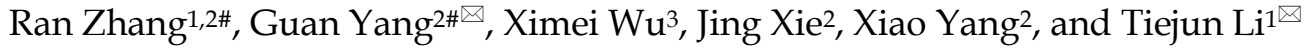 \\ 1. Department of Oral Pathology, Peking University School and Hospital of Stomatology, Beijing, P. R. China; \\ 2. State Key Laboratory of Proteomics, Genetic Laboratory of Development and Diseases, Institute of Biotechnology, Beijing, P. R. China; \\ 3. Department of Pharmacology, Medical College, Zhejiang University, Zhejiang Province, Hangzhou, P. R. China.
}

\# These authors contributed equally to this work.

\begin{abstract}
$\triangle$ Corresponding author: Tiejun Li, DDS, PhD. Department of Oral Pathology, Peking University School and Hospital of Stomatology, 22 South Zhongguancun Avenue, Beijing 100081, PR China. Tel/Fax: 86-10-62179977ext5203(O); E-mail: litiejun22@vip.sina.com, or Guan Yang, Ph.D. Institute of Biotechnology, 20 Dongdajie, Beijing 100071, P.R. China. Tel/Fax: 86-10-66948883(O); E-mail: yogopop3@163.com.
\end{abstract}

(c) Ivyspring International Publisher. This is an open-access article distributed under the terms of the Creative Commons License (http://creativecommons.org/ licenses/by-nc-nd/3.0/). Reproduction is permitted for personal, noncommercial use, provided that the article is in whole, unmodified, and properly cited.

Received: 2012.12.02; Accepted: 2013.01.31; Published: 2013.02.19

\begin{abstract}
Tooth development undergoes a series of complex reciprocal interactions between dental epithelium and the underlying mesenchymal cells. Compared with the study in tooth crown formation, little is known about the molecular mechanism underlying the development of tooth roots. In the present study, we conditionally knock out $\beta$-catenin gene $(C t n n b /)$ within developing odontoblasts and cementoblasts during the development of tooth roots, and observed rootless molars as well as incomplete incisors. Histological analyses revealed intact structure of molar crown and labial side of incisor, however, as for the molar roots and the lingual portion of incisor, the formation of dentin and periodontal tissues were greatly hampered. In situ hybridization experiments using probes of odontoblastic marker genes collagen type l, alpha I (Colla l), osteocalcin $(O C)$ and dentin sialophosphoprotein (Dspp) manifested striking undifferentiation of root odontoblasts in which Ctnnb/ was eliminated. Bromodeoxyuridine (BrdU) labeling and proliferating cell nuclear antigen (PCNA) immunohistochemical experiments also showed retarded proliferation of pre-odontoblasts in mutant mice. However, cell apoptosis was not affected. Additionally, a disrupted formation of cementoblasts, suggested by the absence of transcripts of bone sialoprotein (Bsp) in follicle mesenchyme, was also evident in mutant mice. Our study provides strong in vivo evidence to confirm that $\mathrm{Wnt} / \beta$-catenin signaling is functionally significant to root odontogenesis and cementogenesis during the tooth root development.
\end{abstract}

Key words: Tooth root development, Odontoblast, $\mathrm{Wnt} / \beta$-catenin signaling, mouse model.

\section{Introduction}

Tooth development features a sophisticated series of signaling interactions between the oral epithelium and mesenchyme of neural crest ectomesenchyme origin. Following tooth crown formation, a bilayered epithelial structure termed Hertwig's epithelial root sheath (HERS) was formed by the inner enamel epithelium (IEE) and outer enamel epithelium (OEE) in postnatal teeth. After that, a mutual induction between HERS and root dentin formation occurs. HERS grows in apical direction contouring the future tooth root, and induces odontoblast differentiation from dental papilla to form root dentin; then, the 
odontoblasts signal in turn to regulate the growth and morphogenesis of HERS. During these processes, HERS breaks up into epithelial nests and cords, allowing for dental follicle cells to come in contact with the outer surface of the root and facilitate the formation of periodontium [1, 2]. The developmental model of the lingual side of mouse incisor is similar to that of the molar root, by which HERS and odontoblasts coordinate to generate the enamel-free surface of the lingual incisor [3].

Compared with the knowledge of crown formation, little is known about the molecular regulatory mechanism underlying root development. Gene expression studies have suggested that various signaling pathways might engage in tooth root development, e.g., the interplay between odontoblastic BMP and epithelial Msx is considered to be critical to the tooth root development [4]. Limited studies employing genetically manipulated mouse models have confirmed the in vivo functions of genes during tooth root development. Disrupting Nfic in mice causes striking defects of rootless teeth and malformed incisors [5]. K14-Cre;Smad4f/fl mice, in which dental epithelial TGF- $\beta /$ Smad4 signaling has been inactivated, the formation of odontoblasts, HERS, as well as the molar roots were severely affected [6]. Interestingly, inactivation of Smad4 in odontoblasts can result in retarded maturation of odontoblasts, leading to shortened molar roots and aberrant recession of HERS [7]. However, due to embryonic lethality caused by gene deficiency as well as the lack of Cre transgenic lines that effectively ablate genes within tooth root odontoblasts or HERS, signaling pathways involved in manipulating the formation and development of tooth root still remain largely unknown.

Wnt/ $\beta$-catenin signaling modulates many cellular functions such as proliferation, differentiation and migration, playing important roles in organ development and tissue homeostasis. Binding of Wnt ligands to Frizzled receptors and low-density lipoprotein receptor related protein family co-receptors causes $\beta$-catenin accumulation, nuclear translocation, and transcriptional activation by complexes of $\beta$-catenin and LEF/TCF transcription factor family members [8]. Mice lacking Lef1 exhibited tooth morphogenesis arrest at the bud stage [9]. Epithelium-specific inactivation of $\beta$-catenin or epithelial expression of Dkk1, an inhibitor of canonical Wnt signaling, caused abnormal tooth patterning at the early bud stage $[10,11]$. Interestingly, mesenchyme-specific inactivation of $\beta$-catenin also revealed a critical role of $W n t / \beta$-catenin signaling in the activation of the mesenchymal odontogenic potential during early tooth development [12]. However, it still remains poorly understood how Wnt/ $\beta$-catenin signaling functions during tooth root development at the postnatal stage. Axin2 is a direct target of canonical Wnt signaling [13], previous work employing the Axin2-lacZ reporter mice found an activated $W n t / \beta$-catenin signaling in the region of the developing HERS, dental papilla and the enamel-free lingual side of the incisor (root analogue), indicating canonical Wnt activity in a range of tissues in pre- and postnatal teeth and this activity may play a role in the formation of tooth roots [14]. Nevertheless, direct in vivo evidence is absent up till now.

In order to further understand whether canonical Wnt/ $\beta$-catenin signaling in the tooth mesenchymal cells is of functional importance to tooth root development, we specifically ablated the $\beta$-catenin gene (Ctnnb1), a central component of the canonical Wnt signaling pathway, in odontoblasts, cementoblasts and osteoblasts using the Cre-LoxP system with the previously reported OC-Cre transgenic strain [7]. Our data implied a crucial role of $\mathrm{Wnt} / \beta$-catenin signaling in odontogenesis and cementogenesis, which is indispensable for tooth root development.

\section{Materials and Methods}

\section{Mouse Strains and Genotyping}

Mice homozygous for the floxed Ctnnb1 allele $\left(C t n n b 1^{f l f f}\right)$ were a kind gift from Ximei Wu Laboratory [15], which have two LoxP sites flanking exons 2-6 leading to loss of function deletion. The Ctnnb1-floxed allele mice (Ctnnb1fl/fl, used as wild-type control littermates) were bred with transgenic mice in which the $1.3 \mathrm{~kb}$ mouse Osteocalcin $(O C)$ promoter controls Cre recombinase expression (OC-Cre) within odontoblasts, cementoblasts and osteoblasts. OC-Cre;Ctnnb1flflf (mutant) mice were obtained by breeding the OC-Cre;Ctnnb1f//+ mice with Ctnnb1f/fl mice. For routine genotyping, Cre transgene were detected by PCR using primers described previously [16], and primers for Ctnnb1 locus were designed as follows: forward, $5^{\prime}$-CACCATGTCCTCTGTCTATCC$3^{\prime}$, and reverse, 5'-AAGGTAGAGTGATGAAA GTTGTT-3'. All experimental protocols were designed according to the recommendations of the Beijing Experimental Animal Regulation Board.

\section{Histology, Immunostaining and In situ hybrid- ization}

Six-micrometer sections of mandibles were stained with Sirus red stain by standard methods. The primary antibodies used in immunostaining were performed using primary antibodies against $\beta$-catenin (BD Transduction Laboratories, 610153), keratin 14 
(K14) (Convance, PRB155P-100), bromodeoxyuridine (BrdU) (Abcam, ab6326), proliferating cell nuclear antigen (PCNA) (ZSGB-BIO, ZM-0213), and cleaved caspase-3 (Cell Signaling Technologies, 9661S). Terminal deoxynucleotidyl transferase (TdT)-mediated dUTP nick-end labeling (TUNEL) detection of DNA fragmentation was carried out using a fluorescein-based detection kit (Vazyme, Apoptosis Detection Kit) according to the manufacturer's instructions. Bound antibodies were visualized with diaminobenzidine, Alexa Fluor 488, 594 or TRITC. Sections were counterstained with hematoxylin or DAPI. In situ hybridization of paraffin sections were performed using standard procedures [17]. Digoxigenin-labeled antisense probes were generated from linearized plasmids.

\section{Results}

\section{Ctnnb I was knockout in the odontoblastic layer by OC-Cre transgene}

In the previously reported OC-Cre;ROSA26 double transgenic mice, LacZ expression was first detected in the odontoblasts lined along the tip of the crown in the mandible first molar at postnatal day 1 (P1). At P10, the signals were observed within the original root, but weaker than that in the crown [7]. Full LacZ expression was detected in odontoblasts lining the coronal and root dentin in OC-Cre;ROSA26 mice at P15 (Supplementary Material: Figure S1). We also observed OC-Cre recombinase activity in differentiating cementoblasts outlining the root dentin, which was also reported by another researching group (Supplementary Material: Figure S1, blue arrow indicates)
[18]. Therefore, we employed this OC-Cre strain to specifically ablate floxed genes within developing odontoblasts, cementoblasts and osteoblasts during the tooth root development that begins at P10.

In order to examine whether $\mathrm{Wnt} / \beta$-catenin signaling was activated during tooth root development, we detected the expression of $\beta$-catenin on the P11 and P15 mandible first molar sections using immunofluorescence analysis. In wild-type mice, strong signals could be detected within the pre-odontoblasts and the frontier HERS at the apical portion of tooth root, indicating a key role of $\mathrm{Wnt} / \beta$-catenin signaling in odontogenesis during the initiating step of tooth root morphogenesis (Figure $1 \mathrm{~B}$ and 1D), while in the mutant littermates (OC-Cre;Ctnnb1fl/f), $\beta$-catenin was eliminated within cells throughout the presumptive tooth root position outlined by the HERS (Figure $1 \mathrm{C}$ and $1 \mathrm{E})$. We also investigated the expression of $\beta$-catenin protein on the longitudinal sections of incisors at P15, and observed absence of signals in the lingual side (root analogue) of the mutants as compared with those of the wild-type (Figure $1 \mathrm{~F}$ and $1 \mathrm{G}$ ).

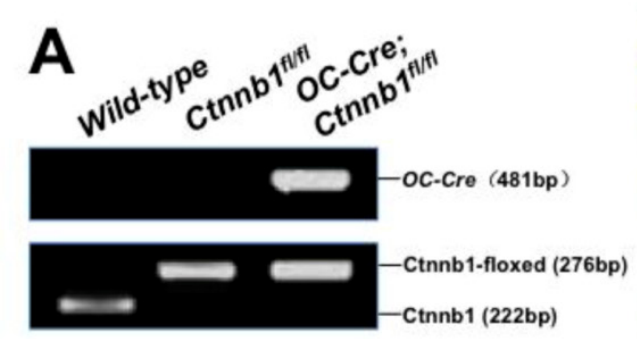

Ctnnb $1^{f / f l}$

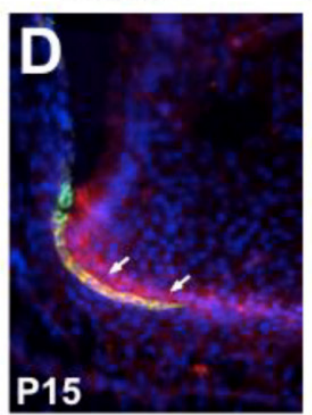
OC-Cre;Ctnnb1 $1^{f / f I}$

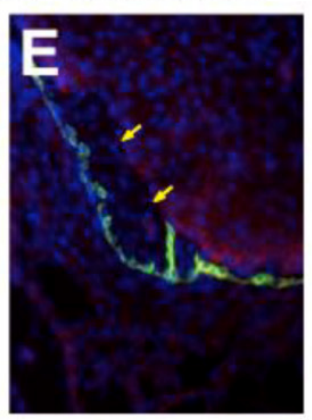

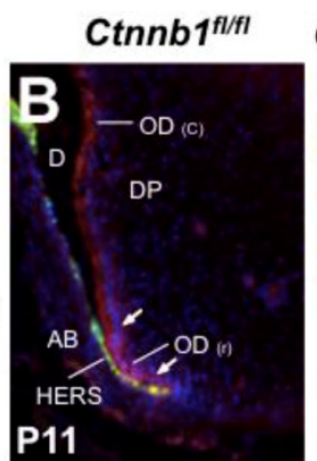
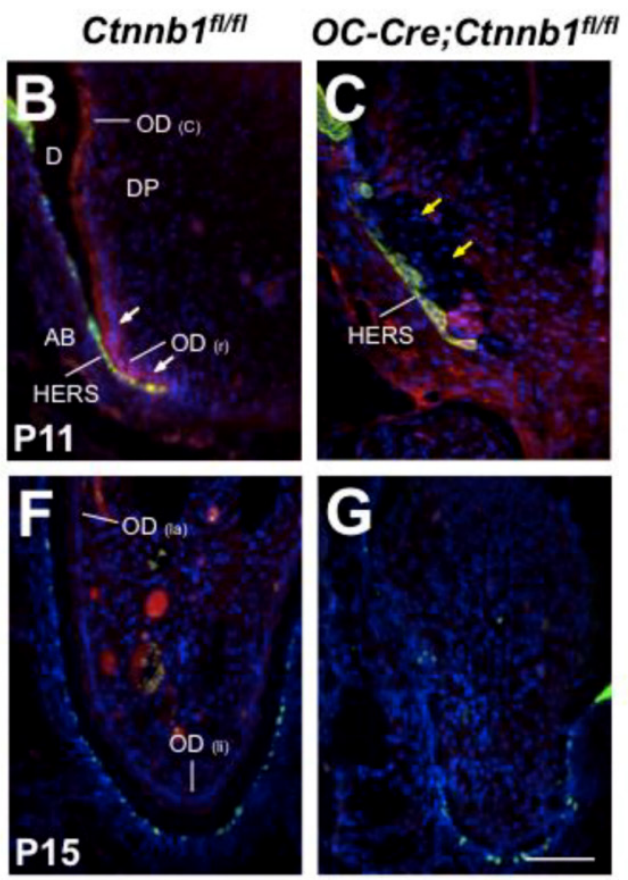

\section{$\beta$-catenin/K14/DAPI}

Figure I. Targeted deletion of $\beta$-catenin in odontoblasts by OC-Cre transgene. (A) Genotyping of Ctnnb If/f and OC-Cre;Ctnnb Iflf $^{f / f}$ mice performed by polymerase chain reaction. Histological sections of the mandible first molar $(B-E)$ and the incisor (F and $G)$ from Ctnnb /f/ff $(B, D$ and F) and OC-Cre;Ctnnb /f/f (C, E and G) mice were stained with antibodies against $\beta$-catenin (red) and KI4 (green). The sections were counterstained with DAPI. Cytoplasmic and nuclear $\beta$-catenin was detected throughout the odontoblasts of the molar and incisor ( $B, D$ and $F$ ), with its highest expression within the pre-odontoblasts and the frontier HERS at the apical portion of molar root (B and D), but in mutant teeth, $\beta$-catenin was absent from the pre-odontoblasts in the apical portion of the molar and in pulp mesenchyme of the incisor (C, E and G). White arrows indicate signals condensed in pre-odontoblasts, and yellow arrows indicate pre-odontoblasts lined along HERS with absence of signals. OD(c), crown odontoblast; OD(r), root odontoblast; D, dentin; DP, dental pulp; AB,

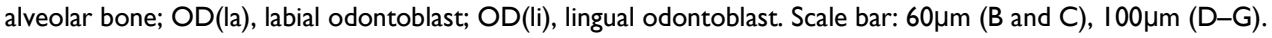




\section{Targeted deletion of $\beta$-catenin in odontoblasts results in rootless molars and incomplete in- cisors.}

The OC-Cre;Ctnnb1f/fl mice were born normally but exhibited smaller body size as they grew up (Figure 2A). According to previous study, OC-Cre;Ctnnb1flfl mice succumbed within 5 weeks [19], however, in our study, as we fed them with soft diet, most of the mutants could survive until P80. Grossly, the OC-Cre;Ctnnb1f/fl incisors were characterized shorter, and were easily worn out in contrast to the wild-type littermates (Figure 2B). A striking abnormality seen in mutant mice was rootless molars. A range of postnatal maxillary molar teeth, from P15 to P60, were extracted and investigated under the stereoscope. The crowns, compared with those of the wild-type, remained relatively unaffected in size and shape. However, the roots of mutant molars seemed completely disappeared (Figure 2C-2F). The P60 rootless molars confirmed that the development of mutant molar roots were arrested rather than delayed.

In order to trace the histological changes between Ctnnb1f/fl and OC-Cre;Ctnnb1flfl teeth, we stained the molar and incisor sections by Sirus red staining (a specific dying protocol for detecting collagen). On P15 wild-type molar sections, apical extension of roots was clearly observed. Root dentin formed smoothly. The periodontal ligament was composed of regularly arranged, woven collagen fibers (Figure 3A). However, on the P15 mutant molar sections, deposition of the root dentin was severely impaired right below the crown. Thin and discrete fibrils were loosely arranged around the presumptive root analogue (Figure 3B). At P45 when wild-type tooth root had already finished development, we could observe a well-developed periodontal structure at the apical portion of wild-type root, with all types of fibers well clustered and regularly arranged, embedding the root inside the alveolar bone (Figure 3E). By contrast, periodontal structure of the mutant molar was poorly developed, the rootless tooth managed to adhere to alveolar bone by connective tissues. Intriguingly, we observed clusters of calcified masses diffused around the presumptive root position (Figure 3F). On the longitudinal sections of mutant incisors at P15 and P45, the dentin of the lingual side was barely detected, with some fragmented calcified tissues left (Figure 3D and $3 \mathrm{H}$ ). Fibrous tissues surrounding the lingual side of the mutant incisors were thinner and looser as compared with those of the wild-type littermates (Figure $3 \mathrm{~d}$ and $3 \mathrm{~h}$ ). These histological data indicated that ablation of Ctnnb1 in developing odontoblasts and cementoblasts resulted in the truncation of tooth root by disturbing the construction of root dentin and periodontium.

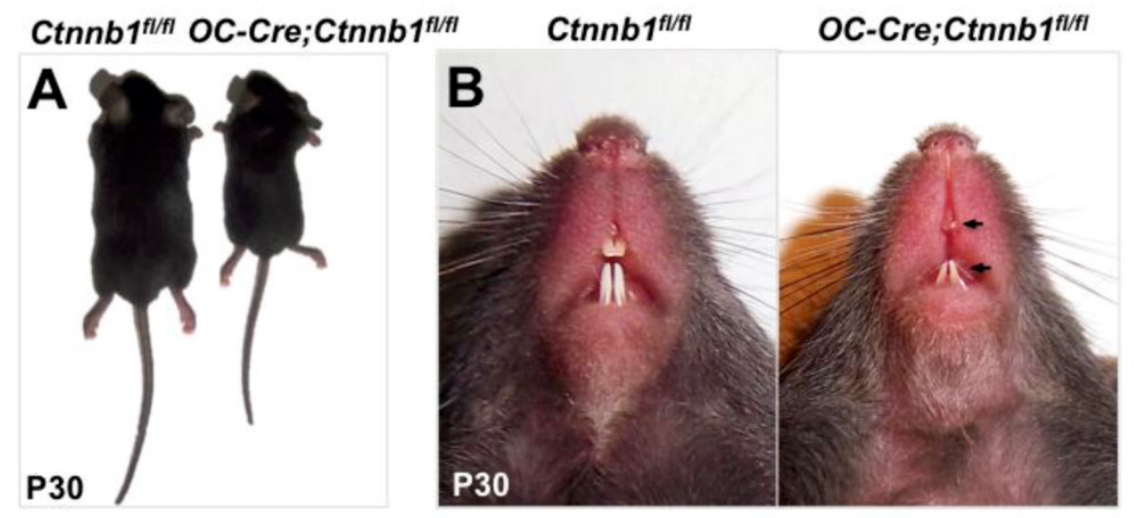

Figure 2. OC-Cre;Ctnnb / f//f mice show growth retardation and abnormalities of tooth root. (A) OC-Cre;Ctnnb If/f mice at I month of age showing shorter stature than the wild-type mice $\left(C t n n b I^{f / f}\right)$. (B) Gross appearance of the incisors at P30. Arrows indicate hypoplastic upper and lower incisors with semitransparent dentin. (C-F) Isolated maxillary first molars of the Ctnnb / fl/fl and OC-Cre;Ctnnb / I/f mice at PI5, P30, P45 and P60. The green and red brackets indicate the lengths of the crown and root, respectively.
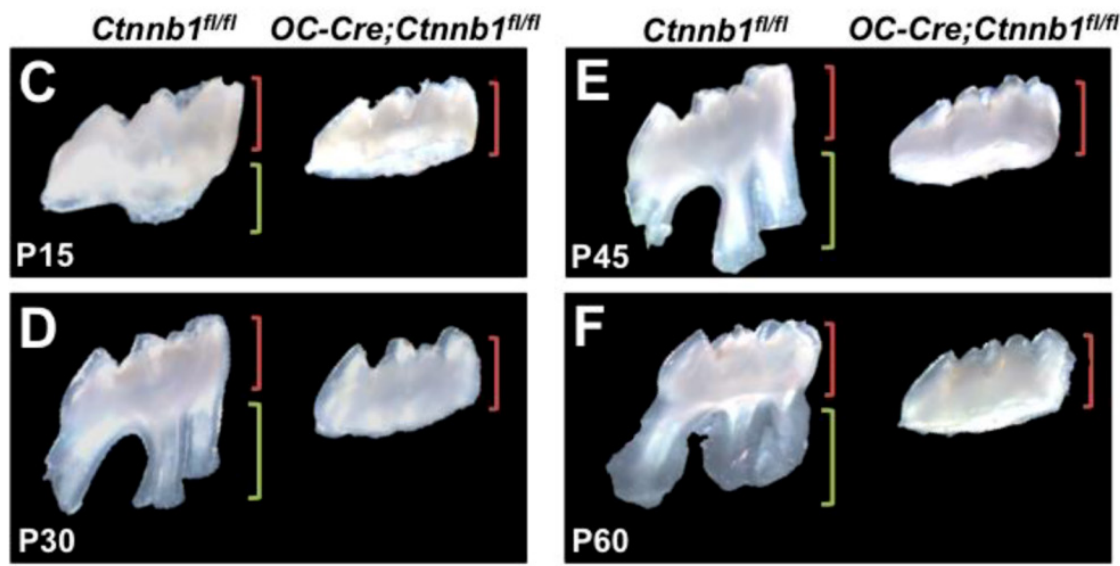

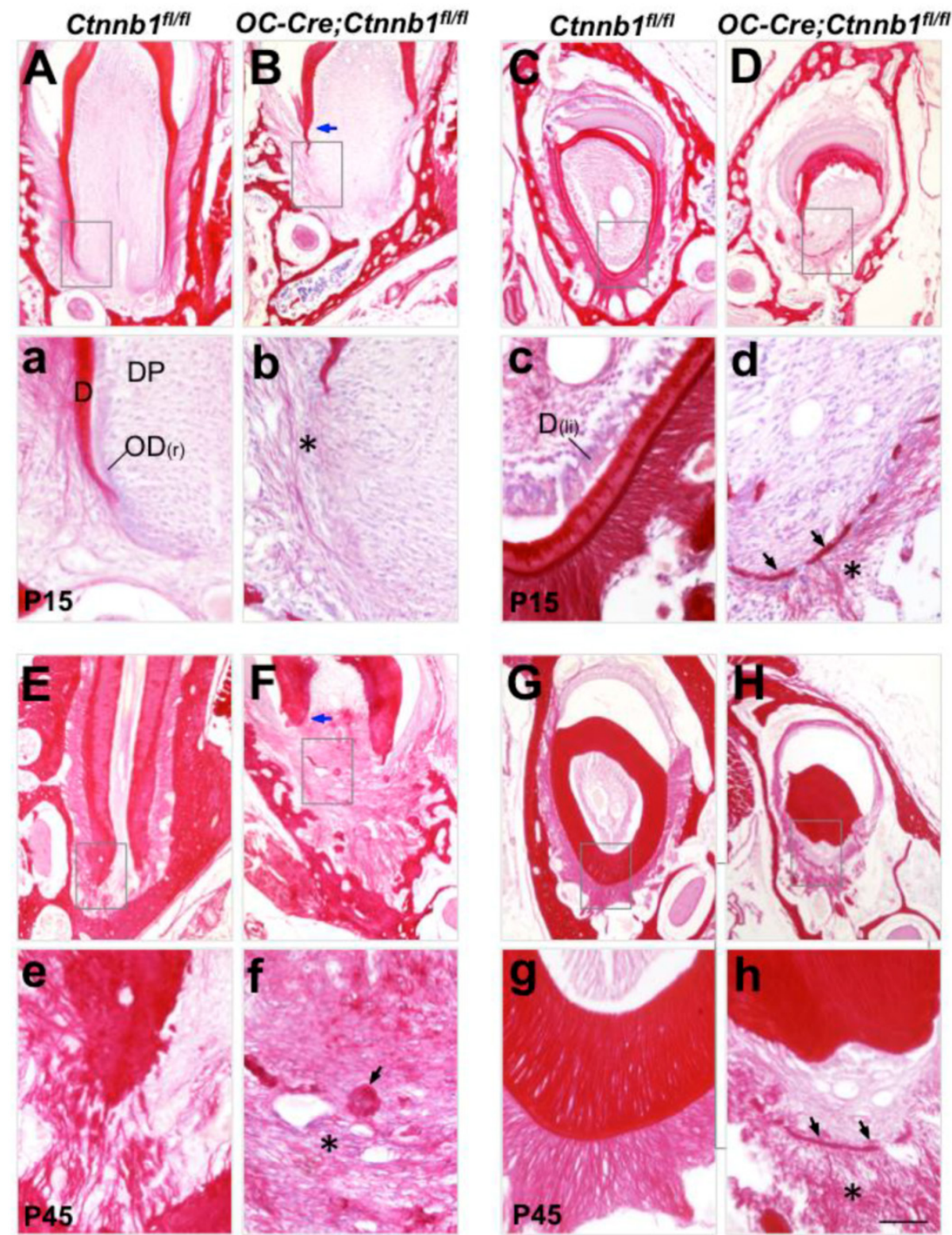

Figure 3. Malformed tooth root development in OC-Cre;Ctnnb $\left.\right|^{f / f}$ mice. Sirus red-stained sections of the mandible first molars (A, B, E and F) and the longitudinal sections of incisors (C, D, G and H) from Ctnnb / fl/f and OC-Cre;Ctnnb $\left.\right|^{f l / f}$ mice at PI5 (A-D) and P45 (E-H). (a-h) The higher-magnification views of the boxed areas in $\mathrm{A}-\mathrm{H}$, respectively. Blue arrows point to the dentin while asterisks denote the periodontal collagen fibrils. On sections from OC-Cre;Ctnnb / I/f teeth, black arrows indicate calcified tissues that were probably deposited by the dysplastic cell bearing Ctnnb/ deficiency. D, dentin; DP, dental pulp; $O D(r)$, root odontoblast; OD(li), lingual odontoblast. Scale bar: $100 \mu \mathrm{m}(\mathrm{A}-\mathrm{H}), 25 \mu \mathrm{m}(\mathrm{a}-\mathrm{h})$

\section{$\beta$-catenin deficiency disrupts the differentia- tion of odontoblasts and cementoblasts}

As the mutant molars failed to develop tooth roots, we then checked over the differentiation status of odontoblasts and cementoblasts by in situ hybridization.

Collagen type I, alpha 1 (Col1a1) can be expressed by odontoblasts, cementoblasts as well as predominant fibroblasts in the periodontal ligament. On wild-type molar sections at P15, transcript signals of Col1a1 were condensed along the differentiating root odontoblasts, whereas in the mutants, signals were almost lost beneath the tooth crown (Figure 4a and 4b). In addition, cells expressing Col1a1 within the periodontium were reduced, implying a compromised collagen synthesis that led to the defected fibrogenesis as seen in Figure 3. In the lingual side of the mutant incisor, the expression of Col1a1 was almost undetectable in odontoblasts whereas decreased in the periodontal cells (Figure 4D). These changes became more evident in mutant mice at P45 (Supplementary Material: Figure S2A-S2D). 

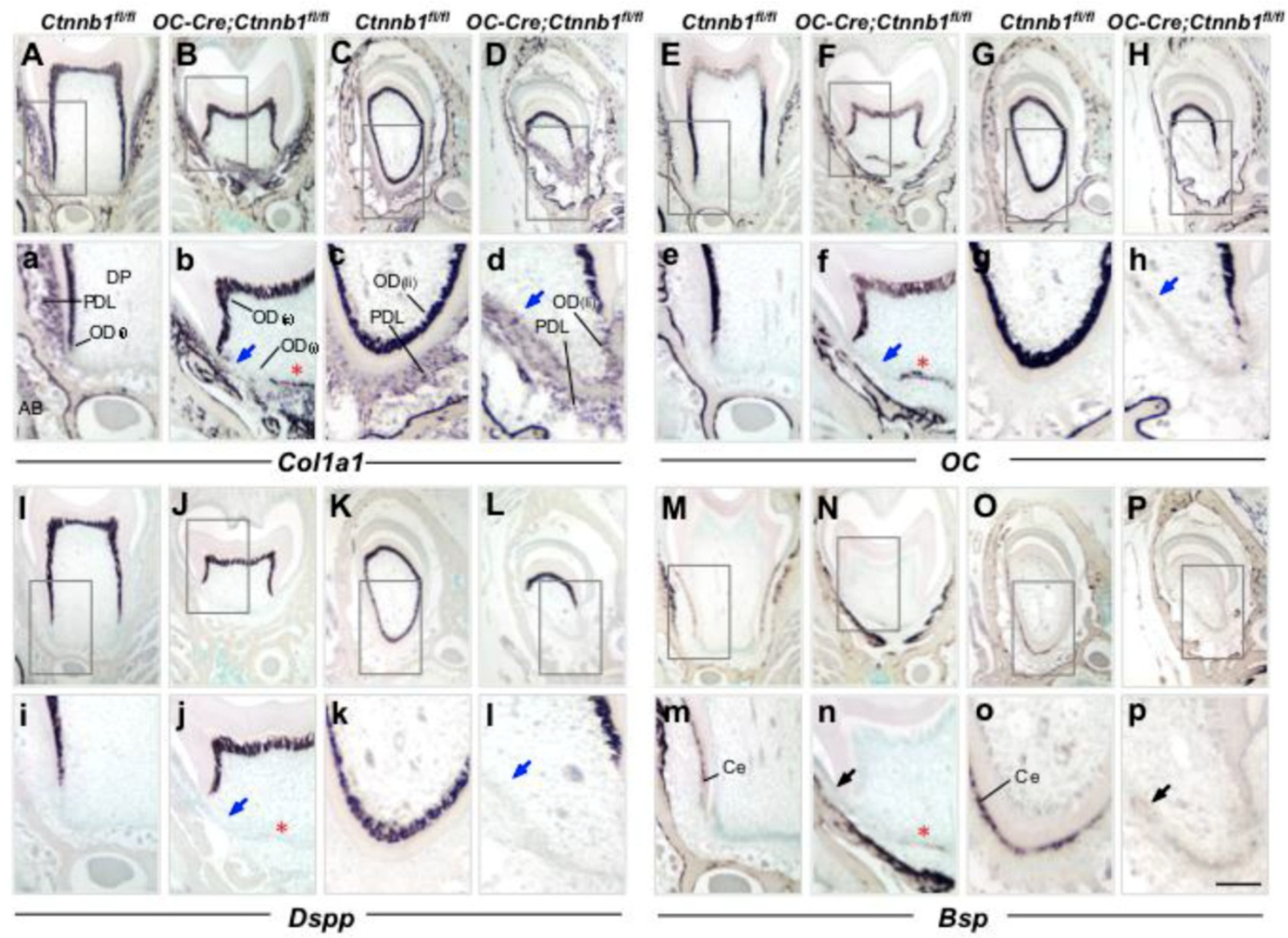

o

p

Bsp

Figure 4. Disruption of $\beta$-catenin in odontoblasts and cementoblasts disrupts their differentiation in OC-Cre; $C$ tnnb /f/f $m$ mice. ISH analyses show the expression of Coll al (A-D), OC (E-H), Dspp (I-L), and Bsp (M-P) on sections of molars and incisors from PI5 Ctnnb If/f and OC-Cre;Ctnnb /fl/f mice. (a-p) The images are higher-magnification views of the boxed areas in the top. In OC-Cre; $C$ tnnb $f^{f / f}$ sections, blue arrows indicate the dysplastic cells beneath the tooth crown and labial incisor that were absent of differential markers for an odontoblastic linage. Red asterisks show that cells lining the calcified tissues were positive for Coll a l, OC and Bsp expression but negative for Dspp expression. Black arrows in $\mathrm{m}-\mathrm{p}$ demonstrate that cementoblasts labeled by Bsp signals were missing within the periodontium of OC-Cre;Ctnnb /f/f teeth. DP, dental pulp; OD(c), crown odontoblast; OD(r), root odontoblast; OD(li), lingual

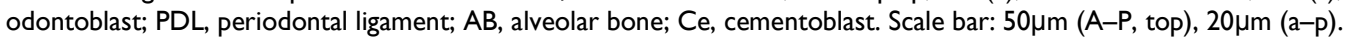

Osteocalcin $(O C)$ is also an early marker gene for odontoblast differentiation, which follows the expression of Col1a1. The absence of OC expression at the presumptive root position in P15 mutants indicated that odontoblastic differentiation had been arrested (Figure 4e and $4 \mathrm{f}$ ). In the root analogue of incisors, absence of $O C$ expression was evident as well (Figure $4 \mathrm{~g}$ and $4 \mathrm{~h}$ ). $O C$ is also a marker gene for cementoblasts, and could be detected outlining the apical dentin of the molar root at P45 (Supplementary Material: Figure S2E). At the similar site of the mutant molar, expression of $O C$ was missing (Supplementary Material: Figure S2F).

Dentin sialophosphoprotein (Dspp), which is a specific marker gene for mature odontoblasts, was also seldom detectable at the presumptive root position in mutant mouse molars as compared to those of the wild-type littermates at P15 and P45, providing the convincing data that root odontogenesis was completely blocked in the context of $\beta$-catenin ablation (Figure 4i and 4j; Supplementary Material: Figure S2I and S2J). In the mutant incisor, similar diminished expression of Dspp could also be seen on the lingual side (Figure 4k and 4l; Supplementary Material: Figure S2K and S2L).

In order to examine whether cementoblasts formation was affected, we employed bone sialoprotein $(B s p)$, a relatively specific marker that could identify cementoblasts lining the outer surface of the root dentin from the surrounding connective tissue (periodontal ligament) region at stages of active cementogenesis $[20,21]$. In wild-type mice, Bsp was specifically presented within the cementoblasts outlining the dentin surface (Figure $4 \mathrm{~m}$ and $4 \mathrm{o}$; Supplementary Material: Figure S2M and S2O). Expression of Bsp was barely detected in neither the mutant molar root nor 
the root analogue of the mutant incisor at P15 and $\mathrm{P} 45$, indicating reduced cementogenesis in mutant mice in contrast to that of the wild-type ones (Figure $4 \mathrm{n}$ and $4 \mathrm{p}$; Supplementary Material: Figure S2N and $\mathrm{S} 2 \mathrm{P})$.

Of interest, some cells between the radicular dental pulp and periodontium were positive for the expression of Col1a1, OC and Bsp, but negative for the expression of Dspp (Figure 4 and Supplementary Material: Figure S2, asterisk indicated). These suspicious cells resided closely to HERS, and were supposed to synthesize and deposit cementoid rather than dentin. However, the cell type and origin need further characterization.

\section{Ctnnb1 $1^{f / f l}$}
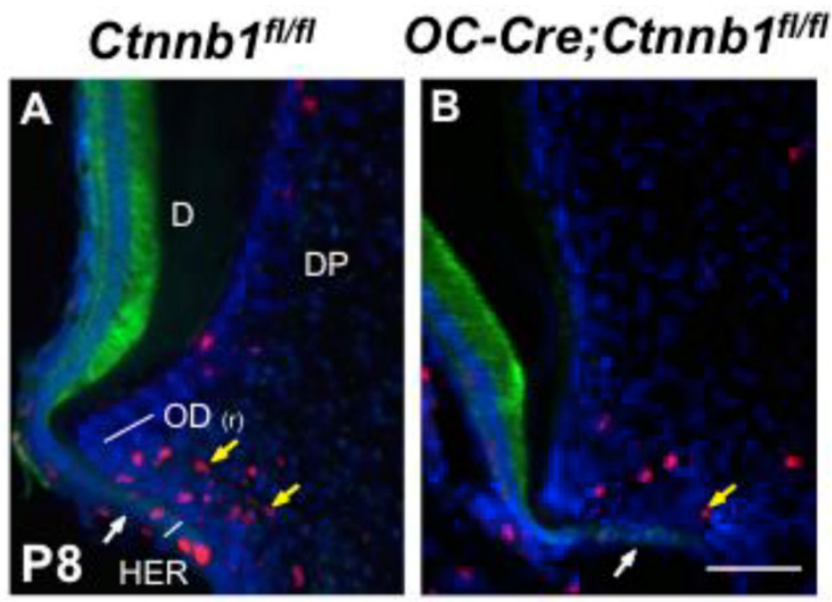

\section{C}

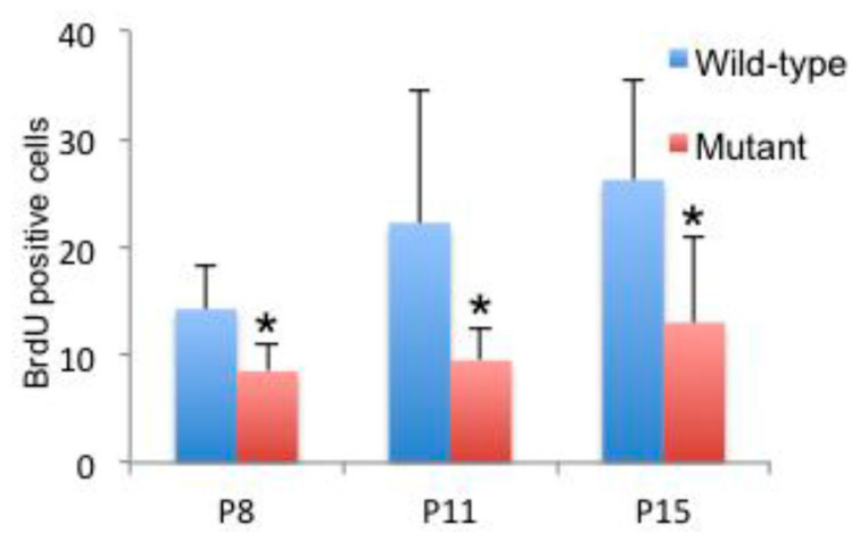

Figure 5. Absence of $\beta$-catenin in odontoblasts impedes their proliferation. (A, B) Double-immunofluorescence of BrdU (red) and KI4 (green) performed on sections of the mandible first molars from P8 Ctnnb $/^{f / f l}$ and OC-Cre;Ctnnb Iflf mice. (C) Quantification of BrdU-positive pre-odontoblasts on sections of the mandible first molars from P8, PI I and PI5 Ctnnb If/fl and OC-Cre;Ctnnb If/fl mice. Yellow arrows indicate that BrdU-positive pre-odontoblasts in OC-Cre;Ctnnb Iflff molars were reduced. White arrows indicate the HERS. D, dentin; DP, dental pulp; OD(r), root odontoblast. Scale bar: $60 \mu \mathrm{m}$ (A and B). Results represent the arithmetic means \pm SEM of results for four mice per group. $*, P<0.05$.

\section{Ctnnb1 ${ }^{f / f l} \quad$ OC-Cre;Ctnnb1 ${ }^{f / f l}$}
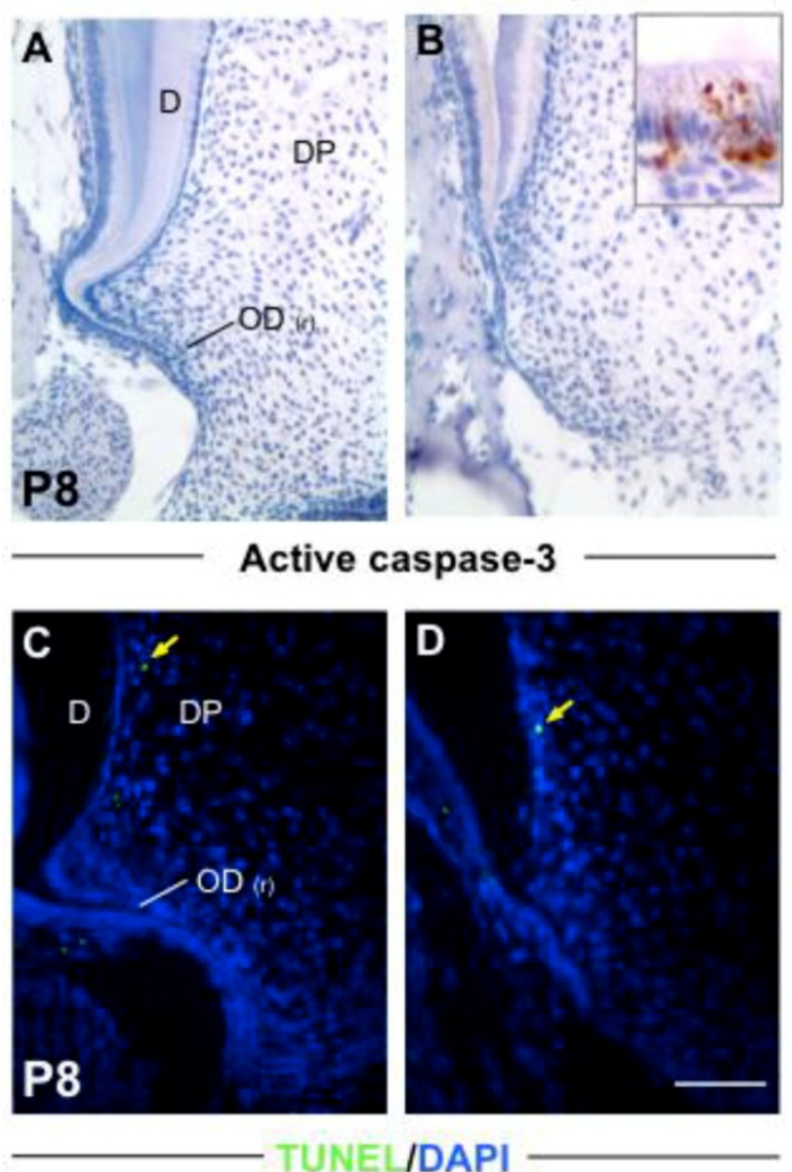

Figure 6. Absence of $\beta$-catenin in odontoblasts has little impact on their apoptosis. Apoptosis was analyzed by active caspase- 3 staining ( $A$ and $B$ ) and TUNEL assay ( $C$ and $D)$. The inset in $B$ shows positive signals in the ameloblast layer as a positive control. Yellow arrows in $C$ and $D$ indicate scarce signals from differentiated odontoblasts. D, dentin; DP, dental pulp; $\mathrm{OD}(\mathrm{r})$, root odontoblast. Scale bar: $100 \mu \mathrm{m}$.

\section{Absence of $\beta$-catenin in odontoblasts retards their proliferation but has no effect on apop- tosis}

In order to accurately map the proliferating cells in the apical part of molars, we double-stained molar sections with K14, which is a specific marker for cells of HERS [2], and with BrdU or PCNA by immunofluorescence. In the wild-type molars at P8 and P15, proliferating cells were condensed around HERS in the radicular portion of tooth roots, revealing a highly proliferating ability of pre-odontoblasts along with tooth root development (Figure 5A and Supplementary Material: Figure S3A). However, in the mutants, less BrdU and PCNA positive cells were detected lining along the fenestrated HERS (Figure $5 \mathrm{~B}$ and Supplementary Material: Figure S3B). Nevertheless, the apoptosis index was not altered between the wild-type and mutant root pre-odontoblasts either by activated caspase- 3 immunohistochemistry or by TUNEL assay (Figure 6A). 
Interestingly, in spite of the absence of root dentin in the mutants, HERS elongated towards the apical direction and successfully fenestrated, outlining the papilla mesenchyme under the crown (Figure 5B and Supplementary Material: Figure S3B). These data indicated that, together with its role in odontoblastic differentiation, intact $\mathrm{Wnt} / \beta$-catenin signaling was required to promote the proliferation of pre-odontoblasts, thus dominating the odontogenesis during tooth root development.

\section{Discussion}

In the present work, ablation of $\beta$-catenin in the odontoblasts and cementoblasts severely disrupted the morphogenesis of the molar roots and the root analogue of incisor. Our data provide strong evidence that the integrity of Wnt/ $\beta$-catenin signaling pathway within odontoblasts is of great importance to cell differentiation and proliferation during tooth root development.

Using our OC-Cre line, we have generated the first conditional mouse model of rootless teeth that can survive after the embryonic and postnatal development. Unlike crown formation, which finished its development in embryo, tooth root development begins postnatally and lasts for almost 3 weeks in mice. Our OC-Cre line enables the spatial and temporal excision of genes exclusively in root pre-odontoblasts and cementoblasts throughout the processes of tooth root development. We previously generated a conditional knockout mouse strain (OC-Cre;Smad4f/ff) in which Smad4 gene was successfully inactivated in odontoblasts, and observed malformed molars with short roots, fractured incisors and irregular dentin in adult mice [7]. However, in contrast to the complete absence of tooth roots in OC-Cre;Ctnnb1/f/fl mice, it's convincing that Wnt/ $\mathrm{B}$-catenin signaling plays a more dominant role than Smad4-mediated pathway during tooth root odontogenesis. Interestingly, rootless molars in OC-Cre;Ctnnb1f/fl mice successfully erupted at around P20 (Figure 3 and data not shown), and were partially functional during the living time. This observation suggested that tooth root might probably be dispensable for tooth eruption. We haven't seen any missing of teeth in mutant mice till they die. The rootless molars attached to the alveolar bone by connective tissues, much similar to the way by which most reptiles and fishes anchor their rootless teeth [2]. We also observed that HERS was normally extended apically in mutant mice, indicating that HERS could form in the absence of root odontoblasts and dentin. However, the molecular mechanisms underlying these interesting phenotypes observed in

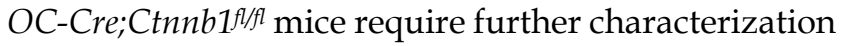
and clarification.
We have provided the first in vivo evidence to prove that deficient $\mathrm{Wnt} / \beta$-catenin signaling blocks odontoblast differentiation during tooth root development. Wnt/ $\beta$-catenin signaling controls cell fate determination in multiple types of tissues by directing the differentiation of progenitor cells. Recently, its functions on differentiation of embryonic stem cells [22], hypothalamic progenitor cells [23], smooth muscle precursors [24], thalamic progenitor cells [25], cardiac progenitor cells [26] and preosteoblasts [27] were revealed by genetic manipulations. Previous research had shown that in the early stage of tooth morphogenesis, mesenchyme-specific inactivation of $\beta$-catenin arrested further tooth development at the bud to cap transition [12], at which odontogenesis still remain uninitiated. As for a tooth root, the most important construction is the fine-deposited dentin produced by root odontoblasts. In OC-Cre;Ctnnb1/f/l mice, $\beta$-catenin was ablated from pre-odontoblasts, resulting in the blockade of odontoblastic differentiation and the reduction of cellular proliferation, thus arrested root dentin synthesis, and ultimately leading to the absence of tooth root. However, in crown odontoblasts, which have finished their differentiation in embryo, following knockout of $\beta$-catenin has no visible effect on their further maturation or maintenance. Consistently, an in vitro study has shown that Wnt/ $\beta$-catenin signaling has a positive stimulating effect on dental papilla stem cells on aspects of proliferation and differentiation, further confirming that $W n t / \beta$-catenin signaling dominates odontogenesis via regulating their odontoblastic differentiation [28]. Interestingly, in another mouse model, constitutive stabilization of $\beta$-catenin in developing odontoblasts leads to abnormal teeth as characterized by shortened roots with odontoblasts acceleratedly differentiated and the resulting excessively deposited dentin [18, 29]. These in vitro and in vivo data, together with our present work, indicates that $\mathrm{Wnt} / \beta$-catenin signaling is of significant importance in tooth root development by promoting the differentiation from mesenchyme to odontoblasts.

\section{Supplementary Material}

Fig.S1 - S3. http://www.ijbs.com/v09p0228s1.pdf

\section{Acknowledgments}

We thank Ximei $\mathrm{Wu}$ for Ctnnb1flfl mice. This study was supported by grants from the State Key Program of National Natural Science of China No. 81030018; the National Basic Research Program of China No. 2012CB966904; the National Natural Science Foundation of China No. 30900863 and No. 81241062 . 


\section{Competing Interests}

The authors have declared that no competing interest exists.

\section{References}

1. Thomas HF. Root formation. Int J Dev Biol 1995; 39: 231-7.

2. Luan X, Ito Y, Diekwisch TG. Evolution and development of Hertwig's epithelial root sheath. Dev Dyn 2006; 235: 1167-80.

3. Tummers M, Thesleff I. Root or crown: a developmental choice orchestrated by the differential regulation of the epithelial stem cell niche in the tooth of two rodent species. Development 2003; 130: 1049-57.

4. Yamashiro T, Tummers M, Thesleff I. Expression of bone morphogenetic proteins and Msx genes during root formation. J Dent Res 2003; 82: 172-6.

5. Steele-Perkins G, Butz KG, Lyons GE et al. Essential role for NFI-C/CTF transcription-replication factor in tooth root development. Mol Cell Biol 2003; 23: 1075-84.

6. Huang $\mathrm{X}, \mathrm{Xu} \mathrm{X}$, Bringas $\mathrm{P}, \mathrm{Jr}$. et al. Smad4-Shh-Nfic signaling cascade-mediated epithelial-mesenchymal interaction is crucial in regulating tooth root development. J Bone Miner Res 2010; 25: 1167-78.

7. Gao Y, Yang G, Weng T et al. Disruption of Smad4 in odontoblasts causes multiple keratocystic odontogenic tumors and tooth malformation in mice. Mol Cell Biol 2009; 29: 5941-51.

8. Logan CY, Nusse R. The Wnt signaling pathway in development and disease. Annu Rev Cell Dev Biol 2004; 20: 781-810.

9. Kratochwil K, Galceran J, Tontsch S et al. FGF4, a direct target of LEF1 and Wnt signaling, can rescue the arrest of tooth organogenesis in Lef1(-/-) mice. Genes Dev 2002; 16: 3173-85.

10. Han XL, Liu M, Voisey A et al. Post-natal effect of overexpressed DKK1 on mandibular molar formation. J Dent Res 2011; 90: 1312-7.

11. Andl T, Reddy ST, Gaddapara T, Millar SE. WNT signals are required for the initiation of hair follicle development. Dev Cell 2002; 2: 643-53.

12. Chen J, Lan $Y$, Baek JA et al. Wnt/beta-catenin signaling plays an essential role in activation of odontogenic mesenchyme during early tooth development. Dev Biol 2009; 334: 174-85.

13. Kim JW, Simmer JP. Hereditary dentin defects. J Dent Res 2007; 86: 392-9.

14. Lohi M, Tucker AS, Sharpe PT. Expression of Axin2 indicates a role for canonical Wnt signaling in development of the crown and root during pre- and postnatal tooth development. Dev Dyn 2010; 239: 160-7.

15. Lin C, Yin Y, Long F, Ma L. Tissue-specific requirements of beta-catenin in external genitalia development. Development 2008; 135: 2815-25.

16. Tan $\mathrm{X}$, Weng $\mathrm{T}$, Zhang J et al. Smad4 is required for maintaining normal murine postnatal bone homeostasis. J Cell Sci 2007; 120: 2162-70.

17. Yang G, Sun Q, Teng Y et al. PTEN deficiency causes dyschondroplasia in mice by enhanced hypoxia-inducible factor 1alpha signaling and endoplasmic reticulum stress. Development 2008; 135: 3587-97.

18. Bae $\mathrm{CH}$, Lee JY, Kim $\mathrm{TH}$ et al. Excessive Wnt/beta-catenin signaling disturbs tooth-root formation. J Periodontal Res 2012.

19. Holmen SL, Zylstra CR, Mukherjee A et al. Essential role of beta-catenin in postnatal bone acquisition. J Biol Chem 2005; 280: 21162-8.

20. MacNeil RL, Berry J, Strayhorn C, Somerman MJ. Expression of bone sialoprotein mRNA by cells lining the mouse tooth root during cementogenesis. Arch Oral Biol 1996; 41: 827-35.

21. D'Errico JA, MacNeil RL, Takata T et al. Expression of bone associated markers by tooth root lining cells, in situ and in vitro. Bone 1997; 20: 117-26.

22. Davidson KC, Adams AM, Goodson JM et al. Wnt/beta-catenin signaling promotes differentiation, not self-renewal, of human embryonic stem cells and is repressed by Oct4. Proc Natl Acad Sci U S A 2012; 109: 4485-90.

23. Wang $X$, Kopinke D, Lin J et al. Wnt signaling regulates postembryonic hypothalamic progenitor differentiation. Dev Cell 2012; 23: 624-36.

24. Trowe MO, Airik R, Weiss AC et al. Canonical Wnt signaling regulates smooth muscle precursor development in the mouse ureter. Development 2012; 139: 3099-108.

25. Bluske KK, Vue TY, Kawakami Y et al. beta-Catenin signaling specifies progenitor cell identity in parallel with Shh signaling in the developing mammalian thalamus. Development 2012; 139: 2692-702.

26. Klaus A, Muller M, Schulz $\mathrm{H}$ et al. Wnt/beta-catenin and Bmp signals control distinct sets of transcription factors in cardiac progenitor cells. Proc Natl Acad Sci U S A 2012; 109: 10921-6.

27. Song L, Liu M, Ono $\mathrm{N}$ et al. Loss of wnt/beta-catenin signaling causes cell fate shift of preosteoblasts from osteoblasts to adipocytes. J Bone Miner Res 2012; 27: 2344-58.

28. Wang C, Ren L, Peng L et al. Effect of Wnt6 on human dental papilla cells in vitro. J Endod 2010; 36: 238-43.
29. Kim TH, Lee JY, Baek JA et al. Constitutive stabilization of ss-catenin in the dental mesenchyme leads to excessive dentin and cementum formation. Biochem Biophys Res Commun 2011; 412: 549-55. 